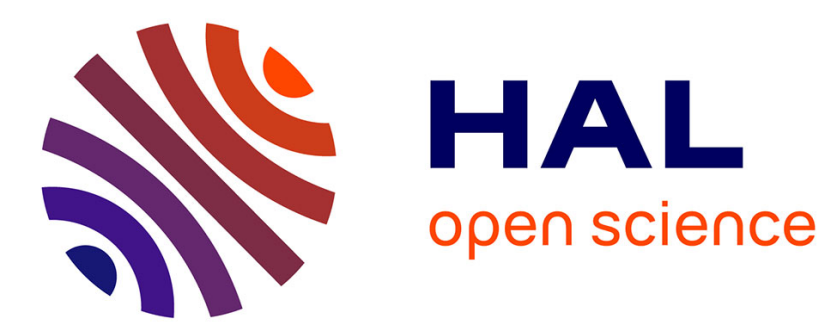

\title{
3D Integral Formulation Using Facet Elements for Thin Conductive Shells Coupled With an External Circuit
}

\author{
Thanh Trung Nguyen, Gérard Meunier, Jean-Michel Guichon, Olivier
}

Chadebec

\section{- To cite this version:}

Thanh Trung Nguyen, Gérard Meunier, Jean-Michel Guichon, Olivier Chadebec. 3D Integral Formulation Using Facet Elements for Thin Conductive Shells Coupled With an External Circuit. IEEE Transactions on Magnetics, 2015, 51 (3), 10.1109/TMAG.2014.2363365 . hal-01157613

\section{HAL Id: hal-01157613 \\ https://hal.science/hal-01157613}

Submitted on 25 Nov 2020

HAL is a multi-disciplinary open access archive for the deposit and dissemination of scientific research documents, whether they are published or not. The documents may come from teaching and research institutions in France or abroad, or from public or private research centers.
L'archive ouverte pluridisciplinaire HAL, est destinée au dépôt et à la diffusion de documents scientifiques de niveau recherche, publiés ou non, émanant des établissements d'enseignement et de recherche français ou étrangers, des laboratoires publics ou privés. 


\title{
3-D Integral Formulation Using Facet Elements for Thin Conductive Shells Coupled With an External Circuit
}

\author{
Thanh-Trung Nguyen, Gérard Meunier, Jean-Michel Guichon, and Olivier Chadebec \\ Grenoble Electrical Engineering Laboratory, Université Grenoble Alpes, Grenoble F-38000, France \\ Grenoble Electrical Engineering Laboratory, Centre National de la Recherche Scientifique, Grenoble F-38000, France
}

\begin{abstract}
A volume integral formulation using facet elements to compute eddy currents in thin conductive shells is presented. The formulation is general and considers the field variation through the depth due to the skin effect. The formulation leads to an equivalent lumped elements circuit that can be coupled with an external circuit. The resolution of the circuit is achieved by the independent loops method. The formulation has been validated because of two numerical examples. Results are compared with an axisymmetric finite-element method (FEM) and another shell formulation implemented in a 3-D FEM.
\end{abstract}

Index Terms - Eddy currents, facet element, generalized partial element equivalent circuit (PEEC) method, integral equation method, thin conductive shells.

\section{INTRODUCTION}

$\mathbf{T}$ HIN conductive shells are characterized by a high ratio between length and thickness. Thus, using a volume mesh for these shells leads to a large number of elements to avoid the numerical errors. Furthermore, when the frequency is high, the skin depth becomes much thinner than the thickness $e$. It increases the size of the needed mesh to properly model this effect.

To compute eddy currents in thin shells in the general case $(\delta>e$ or $\delta \approx e$ or $\delta<e$ ), a few different approaches have already been proposed [1]-[3]. In some of them [1], [2], integral formulations are developed using a boundary integral equation method and or a volume integral equation method. In these formulations, the field variation across the thickness of the shell is considered with a quite good accuracy and the air region is not meshed. In [3], a finite-element formulation using the similar shell element for modeling thin conductive regions has been presented. This formulation considers the field variation through depth due to skin effect and allows the modeling of the multiply connected shells and the coupling with an external electrical circuit but it leads to the mesh of the air region.

The partial element equivalent circuit (PEEC) method is a well-known integral equation technique leading to an equivalent circuit representation of an electromagnetic device [6]. This method is used for the modeling of complex interconnect problems by requiring a structured mesh associated with uniform current density on each element.

Recently, eddy-current integral formulations based on facet elements have been proposed [4], [5]. Different regions have been considered, such as thin regions in [4] and volume regions in [5]. This approach seems very attractive because it enables an easy treatment of the multiply connected problems with general meshes, without cuts technique and meshing the air. Moreover, the coupling with the classical PEEC formulations [6] is natural, since both formulations have the same theoretical bases.

\footnotetext{
Manuscript received June 4, 2014; accepted September 12, 2014 Corresponding author: T.-T. Nguyen (e-mail: thanhtrung.nguyen@g2elab.grenoble-inp.fr).
}

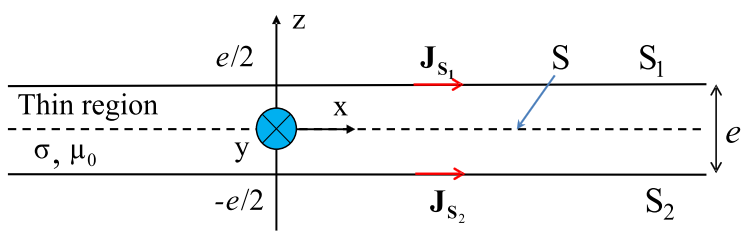

Fig. 1. Both thin shell and surrounding regions are non-magnetic $\left(\mu=\mu_{0}\right)$, the conductivity is $\sigma . S_{1}, S_{2}$ are boundaries of the shell with the air region. $S$ is the equivalent surface region.

This paper presents an integral formulation using facet elements that allows the modeling of non-magnetic conductive thin shells in the general case $(\delta>e$ or $\delta \approx e$ or $\delta<e)$. This approach is similar to [4] but it considers the field variation through depth due to skin effect in low frequency, like in [1]. In our formulation, the current density is linearly interpolated with first-order facet elements. Performances of this formulation will be shown by two examples; the first one is a modeling of the multiply connected region and the second one is a coupling with an external circuit, connected to the thin region.

\section{FORMULATION}

\section{A. Thin Shell Equations}

We consider a non-magnetic shell with a thickness $e$ and a skin depth $\delta$ (Fig. 1).

The current density $\mathbf{J}$ in the region is described by the integral equation of the PEEC method [6]

$$
\frac{\mathbf{J}}{\sigma}+\frac{j \omega \mu_{0}}{4 \pi} \int_{\Omega_{C}} \frac{\mathbf{J}}{r} d \Omega_{C}=-\operatorname{grad} V
$$

where $\sigma$ is the material conductivity, $\mu_{0}$ is the vacuum permeability, and $V$ is the scalar electric potential.

We assume that the current density $\mathbf{J}$ is parallel to the equivalent surface region. Let us define the average shell current $\mathbf{J}_{m}$

$$
\mathbf{J}_{m}=\frac{1}{e} \int_{-e / 2}^{e / 2} \mathbf{J}(z) d z=\frac{G \cdot\left(\mathbf{J}_{\mathbf{S}_{1}}+\mathbf{J}_{\mathbf{S}_{2}}\right)}{2}
$$

with $G=\tanh [(1+j) e / 2 \delta] /[(1+j) e / 2 \delta]$, the characteristics of function $G$ are shown in [7], $\mathbf{J}_{\mathbf{S}_{1}}$ and $\mathbf{J}_{\mathbf{S}_{2}}$ are the current density on both sides of the shell. 
We can write (1) for the first side of the thin region

$$
\frac{\mathbf{J}_{S_{1}}}{\sigma}+\frac{j \omega \mu_{0}}{4 \pi} \int_{\Omega_{C}} \frac{\mathbf{J}}{r} d \Omega_{C}=-\operatorname{grad}_{s} V_{1}
$$

with $V_{1}$ is the scalar electric potential of the first side.

We now consider the following assumption:

$$
\int_{\Omega_{C}} \frac{\mathbf{J}}{r} d \Omega_{C} \approx \int_{S} \frac{1}{r}\left\{\int_{-e / 2}^{e / 2} \mathbf{J}(z) d z\right\} d S=e \int_{S} \frac{\mathbf{J}_{m}}{r} d S .
$$

Using (2)-(4), we finally have

$$
\frac{\mathbf{J}_{S_{1}}}{\sigma}+\frac{j \omega \mu_{0}}{4 \pi} e \int_{S} \frac{\mathbf{J}_{m}}{r} d S=-\operatorname{grad}_{s} V_{1} .
$$

On the second side of the shell, we get a similar equation

$$
\frac{\mathbf{J}_{S_{2}}}{\sigma}+\frac{j \omega \mu_{0}}{4 \pi} e \int_{S} \frac{\mathbf{J}_{m}}{r} d S=-\operatorname{grad}_{s} V_{2}
$$

where $V_{2}$ is the scalar electric potential of the second side.

Averaging equations, which are written for both sides of the shell and using (2), we obtain an integral equation for $\mathbf{J}_{m}$ with $V_{\text {Sm }}=\left(V_{1}+V_{2}\right) / 2$, the average surface potential

$$
\frac{\mathbf{J}_{m}}{G \cdot \sigma}+j \omega \frac{\mu_{0}}{4 \pi} e \int_{S} \frac{\mathbf{J}_{m}}{r} d S=-\operatorname{grad}_{\mathbf{S}} V_{\mathrm{Sm}} .
$$

Thus, we get a thin medium region with the equivalent conductivity $\sigma^{*}=G \cdot \sigma$, the current density $\mathbf{J}_{m}$, the scalar electric potential $V_{\mathrm{Sm}}$.

\section{B. Facet Elements Interpolation}

The average shell current distribution is interpolated with first-order facet elements such as

$$
\mathbf{J}_{m}=\frac{1}{e} \sum_{j} \mathbf{w}_{j} I_{j}
$$

where $I_{j}$ is flux across the $j$ th edge and $\mathbf{w}_{j}$ is the facet shape function with some properties

$$
\begin{aligned}
\mathbf{w}_{j} \cdot \mathbf{n} & = \pm \frac{1}{\ell_{j}} \\
\operatorname{div}_{s} \mathbf{w}_{j} & = \pm \frac{1}{s_{e}}
\end{aligned}
$$

where $\ell_{j}$ is length of the $j$ th edge and $s_{e}$ is surface of element $e$ that contains the edge $j$. The sign $( \pm)$ depends on the facet orientation.

\section{System Assembly Using Facet Elements}

By applying the Galerkin method to (7) and using $\mathbf{w}_{i}$ as projection functions, a system of linear equations is obtained

$$
\left[\mathbf{Z}_{b}\right] \mathbf{I}_{b}=([\mathbf{R}]+j \omega[\mathbf{L}]) \mathbf{I}_{b}=\mathbf{U}_{b}
$$

with

$$
\begin{aligned}
& R_{i j}=\frac{1}{e} \int_{S_{i}} \frac{\mathbf{w}_{i} \cdot \mathbf{w}_{j}}{G \cdot \sigma} d S \\
& L_{i j}=\frac{\mu_{0}}{4 \pi} \int_{S_{i}} \mathbf{w}_{i} \cdot \int_{S_{j}} \frac{\mathbf{w}_{j}}{r} d S d S \\
& U_{\mathrm{bi}}=-\int_{S_{i}} \mathbf{w}_{i} \cdot \operatorname{grad}_{s} V_{\mathrm{Sm}} d S .
\end{aligned}
$$

Like the formulation presented in $[5],\left[\mathbf{Z}_{b}\right]$ can be seen as the impedance matrix of the electrical equivalent circuit generated, $\mathbf{I}_{b}$ is vector of currents through the facets, sparse complex matrix $[\mathbf{R}]$ is the matrix of the resistive terms, and fully dense matrix $[\mathbf{L}]$ is the matrix of the mutual inductances.

Let us apply divergence theorem on (14), we get

$$
U_{\mathrm{bi}}=-\int_{\Gamma}\left(\mathbf{w}_{i} \cdot \mathbf{n}\right) V_{\mathrm{Sm}} d \Gamma+\int_{S} \operatorname{div}_{S}\left(\mathbf{w}_{i}\right) V_{\mathrm{Sm}} d S
$$

where $\Gamma$ is the boundary of surface region $S$.

If edge $i$ is an internal edge

$$
U_{\mathrm{bi}}=\frac{1}{s_{k}} \int_{S_{k}} V_{\mathrm{Sm}} d S_{k}-\frac{1}{s_{l}} \int_{S_{l}} V_{\mathrm{Sm}} d S_{l}
$$

with $s_{k}$ and $s_{l}$ are the surface of element $k$ and $l$ which share the edge $i$. Thus, $U_{\mathrm{bi}}$ is the difference between the averaged voltages of both elements sharing edges $i$.

If the edge $i$ is a border edge, we have

$$
U_{\mathrm{bi}}=-\frac{1}{\ell_{i}} \int_{L_{i}} V_{\mathrm{Sm}} d L_{i}+\frac{1}{s_{e}} \int_{S_{e}} V_{\mathrm{Sm}} d S_{e}
$$

with $s_{e}$ is the surface of element $e$ that contains the edge $i$ and $\ell_{i}$ is length of edge $i$. Thus, $U_{\mathrm{bi}}$ is the difference between the surface averaged voltage on the single elements to whom edge $i$ belongs and the averaged potential on this edge.

From previous considerations, an equivalent electrical circuit (11) can be generated. The branches of this circuit are represented by the edges of initial mesh. Each element of the mesh can then be seen as a node of this circuit. This electrical circuit is in fact an equivalent representation of the dual mesh.

It should be noted in (11) that the resistance matrix [R] is complex; therefore, it cannot be interpreted as purely ohmic components in the equivalent electrical circuit presented.

\section{Resolution of the Electrical Circuit}

To resolve the equivalent electrical circuit, we use the similar technique than which is presented in [5], so using an independent loops search technique [8].

Fundamental circuit equations are expressed with external source voltages

$$
[\mathbf{M}]\left(\mathbf{U}_{b}+\mathbf{U}_{s}\right)=0
$$

with $[\mathbf{M}]$ is the branch-fundamental independent loop transition matrix, where the value of each element can be $-1,0$, or 1 and $\mathbf{U}_{s}$ is the vector of external voltage sources (most part of time equal to 0 ). We can write a new system of linear equations, where unknowns $\mathbf{I}_{m}$ are currents flowing in independent loops

$$
[\mathbf{M}]\left[\mathbf{Z}_{b}\right][\mathbf{M}]^{T} \mathbf{I}_{m}=-[\mathbf{M}] \mathbf{U}_{s} .
$$

Once linear system has been solved, we obtain the currents flowing on each branch by applying the following equation:

$$
\mathbf{I}_{b}=[\mathbf{M}]^{T} \mathbf{I}_{m} .
$$




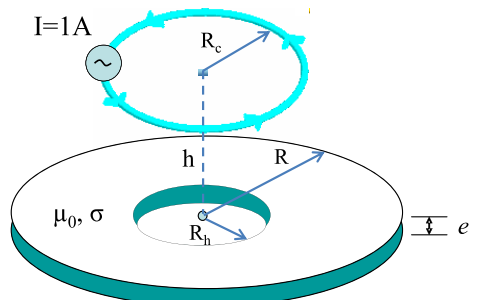

Fig. 2. Thin conductive disk $\left(R=500 \mathrm{~mm}, R_{h}=R / 3, e=10 \mathrm{~mm}\right.$, $\sigma=6 \mathrm{E} 7 \mathrm{~S} / \mathrm{m}, R_{c}=300 \mathrm{~mm}$, and $\left.h=100 \mathrm{~mm}\right)$.

\section{E. Computation of Joule Losses}

Our formulation gives the average shell current after the resolution, which does not allow to calculate the Joules losses integrating the current density $\mathbf{J}(z)$ along the thickness. However, it is possible to evaluate the Joules losses from the expression, like presented in [7]

$$
P=e \cdot \operatorname{Re}\left\{\int_{S} \frac{\mathbf{J}_{m} \cdot \mathbf{J}_{m}^{*}}{G \cdot \sigma} d S-j \omega \int_{S} \frac{\mathbf{B}_{m} \cdot \mathbf{B}_{m}^{*}}{G \cdot \mu_{0}} d S\right\}
$$

where $\mathbf{B}_{m}$ is the tangential component of the total induction $\mathbf{B}_{\text {total, }}$, which can be calculated by the following expression:

$$
\mathbf{B}_{\text {total }}=\frac{G \cdot \mu_{0}}{4 \pi} \int_{S} \frac{\mathbf{J}_{m} \times \mathbf{r}}{r^{3}} d S+\mathbf{B}_{\text {external }}
$$

with $\mathbf{B}_{\text {external }}$ is the induction created by external sources.

\section{NUMERICAL EXAMPLE}

To validate the proposed formulation and to show its performances, we consider two different examples. The first one is a thin conductive disk with a hole placed in a magnetic field created by a current loop (Fig. 2). The second one is a square thin conductive plate connected with a wire, which is supplied by a current source (Fig. 5), presents the coupling of a conductive shell with an external electric circuit. The results of our formulation will be compared with those obtained with flux, a commercial finite-element method (FEM) software.

\section{A. Thin Conductive Disk With a Hole}

In this example, we focus on the computed eddy current distribution and Joules losses in the disk at different frequencies [see Figs. 3(a) and (b) and 4 and Table I]. This example has been modeled by three different methods. The first one is the axisymmetric FEM. The second one is the shell element formulation implemented in 3-D FEM code [3]. The last one is our formulation.

The results provided by our formulation are very encouraging. If we consider the axisymmetric FEM method as our reference, our formulation leads to an error of $0.87 \%$ for the Joules losses at $10 \mathrm{~Hz}(e / \delta=0.49), 1.10 \%$ at $50 \mathrm{~Hz}(e / \delta=1.08)$, and $0.21 \%$ at $250 \mathrm{~Hz}(e / \delta=2.43)$. Let us notice that our formulation leads to more accurate results than the same shell model but coupled with $3-\mathrm{D}$ T- $\varphi$ FEM [3] with 2000000 elements (400000 shell elements in the disk) (Fig. 4).

Another advantage of our formulation is its capacity to treat any multiply connected problems because of the algorithm detecting the independent loops.

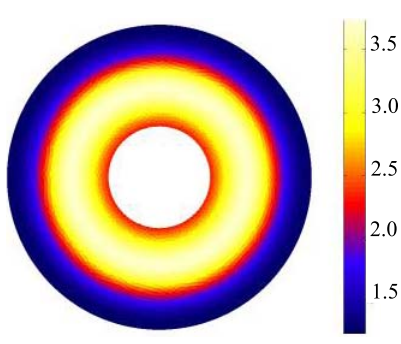

(a)

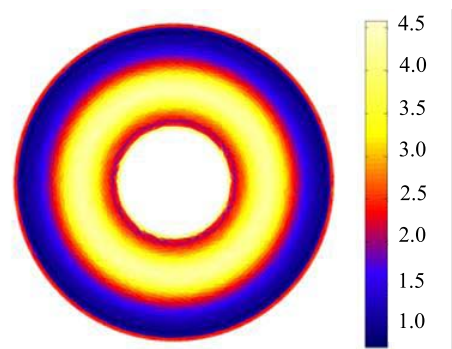

(b)
Fig. 3. Shell current distribution (ampere per meter) in disk at (a) 10 and (b) $250 \mathrm{~Hz}$

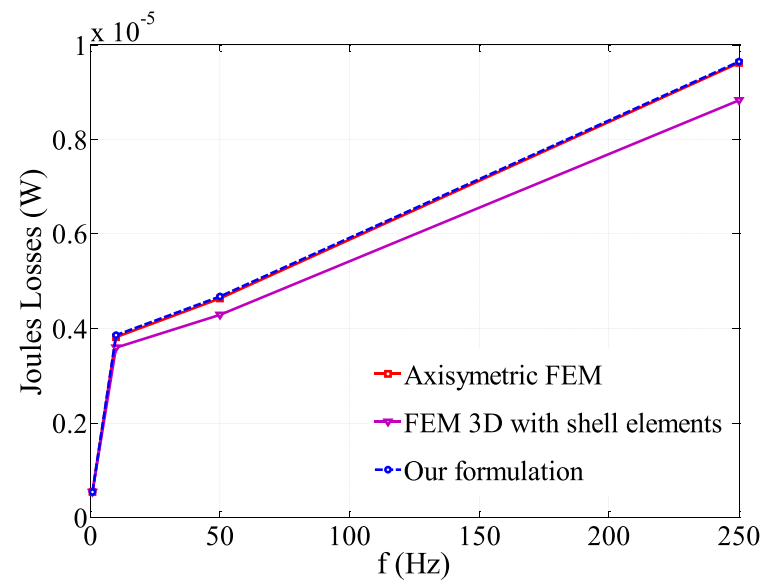

Fig. 4. Joule losses in the disk, calculated by three methods versus frequency.

TABLE I

RELATIVE ERROR OF OUR FORMULATIONS COMPARED WITH THE AXISYMMETRIC FEM SOLUTION

\begin{tabular}{|c|c|c|c|c|}
\hline $\mathrm{f}$ & $e / \delta$ & $\begin{array}{c}\text { Joules Losses (W) } \\
\text { computed by our } \\
\text { formulation } \\
(9000 \text { elements })\end{array}$ & $\begin{array}{c}\text { Joules Losses (W) } \\
\text { computed by } \\
\text { axisymmetric FEM } \\
(90000 \text { elements })\end{array}$ & $\begin{array}{c}\text { Diff. } \\
(\%)\end{array}$ \\
\hline 10 & 0.49 & $3.85 \mathrm{E}-6$ & $3.81 \mathrm{E}-6$ & 0.87 \\
\hline 50 & 1.08 & $4.68 \mathrm{E}-6$ & $4.62 \mathrm{E}-6$ & 1.10 \\
\hline 250 & 2.43 & $9.64 \mathrm{E}-6$ & $9.62 \mathrm{E}-6$ & 0.21 \\
\hline
\end{tabular}

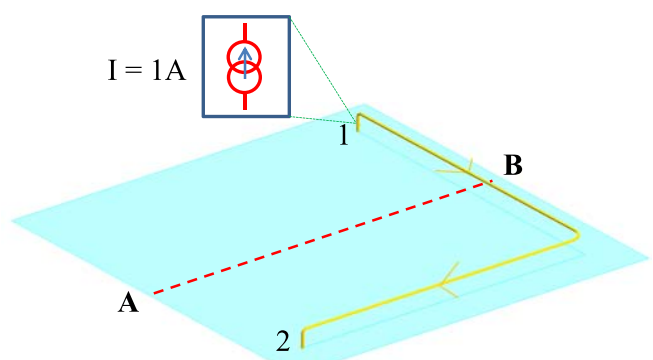

Fig. 5. Aluminum wire connected to a square thin plate conductive in which supplied by a current source, $e=2 \mathrm{~mm}$, length $=200 \mathrm{~mm}$, $\rho=2.836 \times 10^{-8} \Omega \mathrm{m}$, point $A(0,-98,0)$, and point $B(0,98,0)$.

\section{B. Thin Conductive Plate and Wire}

The second example can be seen as the coupling of our formulation with the classical PEEC method [6]. The currents in the square thin plate conductive have been modeled with our formulation and those in the wire have been modeled by the PEEC method. Using the facet elements and the 


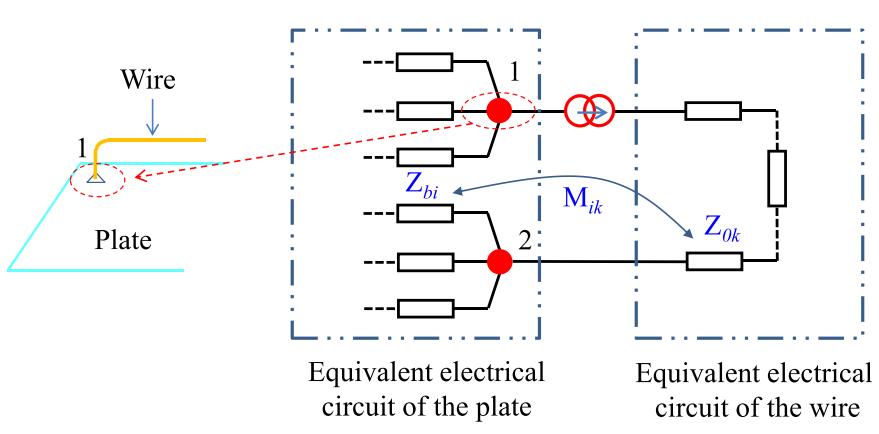

Fig. 6. Global equivalent electrical circuit coupling facet integral and PEEC approaches $\left(Z_{\mathrm{bi}}\right.$ is the impedance of the $i$ th branch of the equivalent electrical circuit of the plate, $\mathrm{Z}_{0 k}$ is the impedance of the $k$ th branch of the equivalent electrical circuit of the wire, and $M_{\mathrm{ik}}$ is the mutual between these two branches).

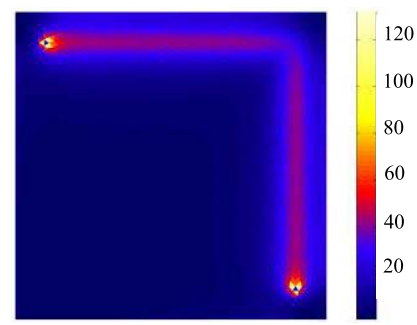

(a)

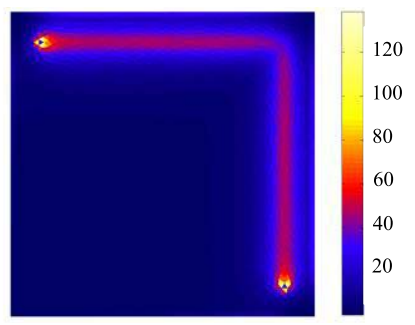

(b)
Fig. 7. Shell current distribution (ampere per meter) in the plate obtained by our formulation at (a) $1 \mathrm{KHz}$ and (b) $1 \mathrm{MHz}$.

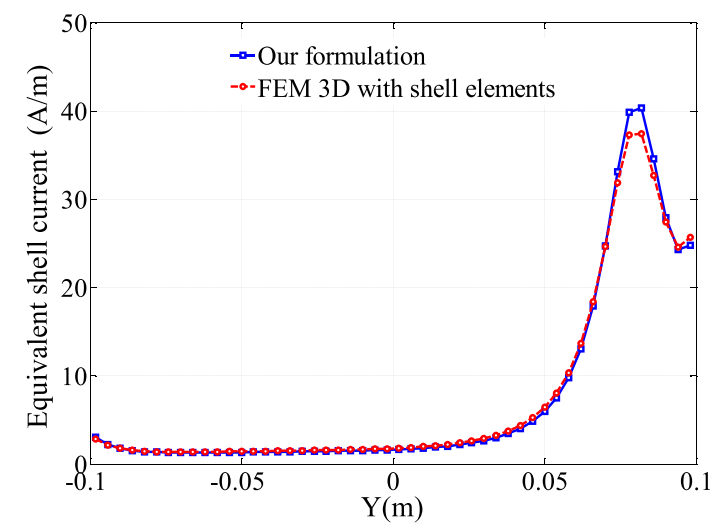

Fig. 8. Shell current distribution along path $\mathrm{AB}$ computed by both formulations at $1 \mathrm{KHz}$.

PEEC method, we obtained the different parts of the global equivalent electrical circuit (Fig. 6). The results are obtained by the resolution of the complete global circuit.

In Fig. 6, the mutual between the $i$ th branch of the equivalent electrical circuit of the plate and the $k$ th branch of the equivalent electrical circuit of the wire $\left(M_{\mathrm{ik}}\right.$ and $\left.M_{\mathrm{ki}}\right)$ are calculated by the expression

$$
\begin{aligned}
M_{\mathrm{ik}} & =\frac{j \omega \mu_{0}}{4 \pi} \int_{S_{i}} \mathbf{w}_{i} \cdot \int_{\Omega_{0 k}} \frac{\mathbf{u}_{k}}{S_{0 k} \cdot r} d \Omega_{0} d S \\
M_{\mathrm{ki}} & =\frac{j \omega \mu_{0}}{4 \pi} \int_{\Omega_{0 k}} \frac{\mathbf{u}_{k}}{S_{0 k}} \cdot \int_{S_{i}} \frac{\mathbf{w}_{i}}{r} d S d \Omega_{0}
\end{aligned}
$$

where $\Omega_{0}$ is the wire region, $\mathbf{u}_{k}$ is the unit vector defining the current direction in the wire, and $S_{0 k}$ is the section of element $k$ of the wire conductor.
TABLE II

Joule LosSES IN THE Plate (w)

\begin{tabular}{|c|c|c|c|}
\hline $\mathrm{f}$ & $e / \delta$ & $\begin{array}{c}\text { Our formulation } \\
(6,500 \text { elements })\end{array}$ & $\begin{array}{c}\text { FEM 3D with shell elements } \\
(2,000,000 \text { elements })\end{array}$ \\
\hline $1 \mathrm{kHz}$ & 0.74 & $8.746 \mathrm{E}-3$ & $8.392 \mathrm{E}-3$ \\
\hline
\end{tabular}

This example is modeled by our formulation and the shell element formulation implemented in 3-D FEM. We still focus on the computed eddy current distribution and Joule losses in the plate.

Simulations have been performed at different frequencies from $10 \mathrm{~Hz}$ to $1 \mathrm{MHz}$. However, only results of simulations at $1 \mathrm{KHz}$ are shown (see Figs. 7 and 8 and Table II).

The results show that the Joule losses calculated by our formulation present a difference of $4.21 \%$ compared with those obtained with shell element formulation in 3-D FEM at $1 \mathrm{KHz}$. Current distributions present a good adequacy.

\section{CONCLUSION}

In this paper, we have presented an original integral volume formulation using facet elements to model thin conductive and non-magnetic regions. The formulation is general and various skin depths $(\delta>e$ or $\delta \approx e$ or $\delta<e)$ are considered.

The formulation has a small relative error in comparison with an axisymmetric reference FEM. Cuts technique and meshing the air are not necessary for solving multiply connected problems. Moreover, it enables an easy and natural treatment of the coupling with the classical PEEC formulation both formulations having the same theoretical bases.

The obtained matrix fully dense but this problem can be overcome by the use of a compression technique like fast multi-pole method [9] enabling the save of memory and the reducing the computation time.

\section{REFERENCES}

[1] L. Krähenbühl and D. Muller, "Thin layers in electrical engineeringexample of shell models in analysing eddy-currents by boundary and finite element methods," IEEE Trans. Magn., vol. 29, no. 2, pp. 1450-1455, Mar. 1993.

[2] T. Le-Duc, G. Meunier, O. Chadebec, and J.-M. Guichon, "A new integral formulation for eddy current computation in thin conductive shells," IEEE Trans. Magn., vol. 48, no. 2, pp. 427-430, Feb. 2012.

[3] C. Guérin and G. Meunier, "3-D Magnetic scalar potential finite element formulation for conducting shells coupled with an external circuit," IEEE Trans. Magn., vol. 48, no. 2, pp. 323-326, Feb. 2012.

[4] P. Alotto, F. Desideri, F. Freschi, A. Machio, and M. Reptto, "Dual-PEEC modeling of a two-port TEM cell for VHF applications," IEEE Trans. Magn., vol. 47, no. 5, pp. 1486-1489, May 2011.

[5] T.-T. Nguyen, G. Meunier, J.-M. Guichon, O. Chadebec, and T.-S. Nguyen, "An integral formulation for the computation of 3-D eddy current using facet elements," IEEE Trans. Magn., vol. 50, no. 2 , pp. 549-552, Feb. 2014, Art. ID 7013504.

[6] A. E. Ruehli, "Equivalent circuit models for three-dimensional multiconductor systems," IEEE Trans. Microw. Theory Techn., vol. 22, no. 3 , pp. 216-221, Mar. 1974.

[7] L. Krähenbühl, P. Dular, T. Zeidan, and F. Buret, "Homogenization of lamination stacks in linear magnetodynamics," IEEE Trans. Magn., vol. 40, no. 2, pp. 912-915, Mar. 2004.

[8] T.-S. Nguyen, J.-M. Guichon, O. Chadebec, G. Meunier, and B. Vincent, "An independent loops search algorithm for solving inductive PEEC large problems," Prog. Electromagn. Res. M, vol. 23, pp. 53-63, Jan. 2012.

[9] T.-S. Nguyen, J.-M. Guichon, O. Chadebec, P. Labie, and J.-L. Coulomb, "Ships magnetic anomaly computation with integral equation and fast multipole method," IEEE Trans. Magn., vol. 47, no. 5, pp. 1414-1417, May 2011. 